\title{
Die sächsische NPD: Politische Struktur und gesellschaftliche Verwurzelung
}

\author{
Marc Brandstetter
}

Das Ergebnis der sächsischen Landtagswahl vom 19. September 2004 versetzte die Bundesrepublik in Angst und Schrecken. Die NPD bekam fast aus dem Stand 9,2 Prozent der abgegebenen Stimmen und zog erstmals seit 36 Jahren wieder in einen Landtag ein. ${ }^{1}$ Nur zwei Jahre nach diesem Überraschungserfolg gelang sogar eine Wiederholung des Triumphes, als die rechtsextreme Partei mit einem ebenfalls stattlichen Ergebnis von 7,3 Prozent eine Fraktion ins Schweriner Schloss entsenden konnte. ${ }^{2}$ Ausgehend von diesen Ereignissen möchte dieser Beitrag die politische Struktur des NPD-Landesverbands Sachsen analysieren und der Frage nachgehen, ob es der Partei zukünftig gelingen kann, weitere Erfolge zu erzielen. Darf demnach Sachsen ein Modellcharakter für die Entwicklung einer rechtsextremen Erfolgsstrategie zugeschrieben werden, und müssen wir mit einer aufsteigenden Tendenz des parteipolitisch organisierten Rechtsextremismus über die Grenzen des Freistaates hinaus rechnen?

Das gute Wahlergebnis der NPD legt die Vermutung nahe, dass es sich bei den Wählern nicht ausschließlich um Personen aus dem rechtsextremen Milieu gehandelt hat. Ist es der NPD - zumindest in diesem Bundesland - gelungen, sich aus einer Außenseiterposition heraus in die „Mitte der Gesellschaft“ zu integrieren? Und hinsichtlich der Mitglieder- und Wählerstruktur ist zu fragen, ob sich diese im Vergleich zur Gründungs- und Frühphase der Partei verändert haben. Darüber hinaus müssen auch die parteieigenen Strukturen, Strategien sowie das Image vor Ort untersucht werden. Ist es etwa gerechtfertigt, im Zusammenhang mit der Wählerklientel der Partei im Osten der Bundesrepublik von der Herausbildung eines eigenen Wählermilieus zu sprechen? Führen die Wahlerfolge zu einer Stabilisierung der Partei über die Grenzen des Landes Sachsen hinaus, vielleicht sogar in der gesamten Bundesrepublik?3

\section{1. „Sonderfall“ Sachsen - Brückenkopft auf dem Weg zur Macht?}

\subsection{Der NPD-Landesverband Sachsen: Infrastruktur und politische Bedeutung}

In den letzten Jahren war die NPD in Sachsen einer starken Mitglieder-Fluktuation unterworfen. Das Jahr 1998 markierte mit 1.400 in der Partei organisierten Personen den bisherigen Höchststand, nur fünf Jahre später hatte sich die Mitgliederzahl allerdings fast

1 Vgl. Eckhard Jesse, Die sächsische Landtagswahl vom 19. September 2004: Debakel für CDU und SPD gleichermaßen, in: ZParl, 36. Jg. (2005), H. 1, S. $80-100$.

2 Vgl. Nikolaus Werz / Steffen Schoon, Die mecklenburg-vorpommersche Landtagswahl vom 17. September 2006: Ein halber Regierungswechsel und das Ende des Dreiparteiensystems, in: ZParl, 38. Jg. (2007), H. 1, S. $67-83$.

3 Vgl. Marc Brandstetter, Die NPD im 21. Jahrhundert. Eine Analyse ihrer aktuellen Situation, ihrer Erfolgsbedingungen und Aussichten, Marburg 2006.

4 Zitiert nach Frankfurter Allgemeine Zeitung vom 7. Dezember 2004. 
halbiert (2003: 800 Mitglieder) ${ }^{5}$, demnach lebte seinerzeit fast ein Drittel aller NPD-Mitglieder im Freistaat. ${ }^{6}$ Nach dem Einzug in den Landtag hat die Partei den starken Mitgliederschwund zunächst stoppen können; es ist offensichtlich sogar gelungen, den Trend umzukehren. ${ }^{7}$ Der Verfassungsschutz schätzt, dass derzeit ungefähr 1.000 Personen eine Parteimitgliedschaft unterschrieben haben, damit ist Sachsen zur größten Landesgliederung in der Bundesrepublik herangewachsen. ${ }^{8}$

Auch an der Anzahl der Kreisverbände ist die überdurchschnittliche organisatorische Stärke der sächsischen NPD zu erkennen; mit 26 Kreisverbänden ist sie nahezu flächendeckend vertreten. ${ }^{9}$ Durch die Gründungen der Kreisverbände Aue-Schwarzenberg, Chemnitz, Mittlerer Erzgebirgskreis, Vogtland und Weißeritzkreis konnte die Organisationsdichte sogar noch ausgebaut werden. ${ }^{10}$ Mit dem Umzug weiterer Parteistrukturen verfestigte die NPD diese herausragende Position: 1999 siedelte die Parteizeitung „Deutsche Stimme“ (DS) von Bayern nach Riesa um ${ }^{11}$, wenig später verlegte auch die Jugendorganisation Junge Nationaldemokraten (JN) ihren Sitz dorthin. ${ }^{12}$

Begünstigt wurde der Aufstieg zum größten NPD-Landesverband zusätzlich durch das gezielte Werben um ehemalige Kader verbotener Neonazi-Organisationen in den frühen neunziger Jahren. Die Möglichkeit, in der NPD in Führungspositionen aufzusteigen, zog neben „Szene-Spitzenleuten“ ebenso Mitglieder der Basis an. ${ }^{13}$ Darüber hinaus organisierte die Partei Veranstaltungen, die sich direkt an rechtsextreme Skinheads wandten. ${ }^{14}$

\subsection{Wahlteilnahme in Sachsen}

Zunächst ging die organisatorische Entfaltung der NPD nicht mit einer gesteigerten Akzeptanz bei Wahlen einher. Bei der Bundestagswahl 1998 machte die Partei mit 1,2 Prozent der Stimmen erstmals über die Landesgrenzen hinaus auf sich aufmerksam. ${ }^{15}$ Bei der ein Jahr später stattfindenden Landtagswahl entfielen bereits 1,4 Prozent der Stimmen auf sie. ${ }^{16}$

5 Vgl. Sächsisches Staatsministerium des Inneren, Verfassungsschutzbericht 2003, Dresden 2004, S. $32 \mathrm{f}$.

6 Vgl. Armin Pfahl-Traughber, Der ,zweite“ Frühling der NPD zwischen Aktion und Politik, in: Uwe Backes / Eckhard Jesse (Hrsg.), Jahrbuch Extremismus \& Demokratie (E \& D), 11. Jg., Baden-Baden 1999, S. 146 - 166, S. 159.

7 Vgl. Homepage des Landesamtes für Verfassungsschutz Sachsen, 15 Jahre NPD in Sachsen, http://www.sachsen.de/de/bf/verwaltung/verfassungsschutz/aktuelles/index.html [10. Juli 2005].

8 Vgl. Sächsisches Staatsministerium des Inneren, Verfassungsschutzbericht 2005 (Kurzfassung), Dresden 2006, S. 9, http://www.sachsen.de/de/bf/verwaltung/verfassungsschutz/berichte/index. html [12. März 2006].

9 Vgl. Homepage des Landesamtes für Verfassungsschutz Sachsen, http://www.sachsen.de/de/bf/ verwaltung/verfassungsschutz/extremismus/index.html [6. März 2007].

10 Vgl. Sächsisches Staatsministerium des Inneren, Verfassungsschutzbericht 2005, Dresden 2006, S. $9 \mathrm{f}$.

11 Vgl. Süddeutsche Zeitung vom 24. Januar 2005.

12 Vgl. Bundesministerium des Innern, Verfassungsschutzbericht 2003, Berlin 2004, S. 68.

13 Vgl. Süddeutsche Zeitung vom 24. Januar 2004.

14 Vgl. Sächsischer Landtag, Drucksache 3/10738, 2004, S. 4.

15 Vgl. Armin Pfahl-Traughber, a.a.O., S. 159.

16 Vgl. Infratest dimap (Hrsg.), Wahlreport. Sachsen hat gewählt. Landtagswahl in Sachsen 19. September 2004, Berlin 2004, S. 21. 
Im Jahr 2004 schließlich konnte die NPD nach fast 36 Jahren währender Abwesenheit endgültig auf eine landespolitische Bühne zurückkehren. Angedeutet hatte sich diese Entwicklung bereits bei der Wahl zum Europäischen Parlament im Juni des Jahres, als die NPD mit 3,3 Prozent in Sachsen ihr bundesweit bestes Resultat erzielen konnte. In sechs Kreisen votierten sogar mehr als fünf Prozent der Wähler für die NPD. ${ }^{17}$ Die zeitgleich stattfindenden Kommunalwahlen bestätigten diesen Trend: Die NPD konnte in fünf Kreistage $^{18}$ und 14 Stadträte einziehen; herauszuheben sind dabei Ergebnisse in Königstein (21,1 Prozent) und Sebnitz (13,2 Prozent). Das spektakulärste Resultat erzielte sie indes bei der Wahl zu einem Gemeindeparlament: In Rheinhardsdorf-Schöna votierte mehr als ein Viertel der Bevölkerung für die NPD (25,2 Prozent). Insgesamt konnte die Partei ihre Sitze auf lokaler Ebene mehr als vervierfachen. Entfielen bei den Kommunalwahlen 1999 neun Sitze auf die NPD, waren es fünf Jahre später $44 .{ }^{19}$

Der endgültige Durchbruch gelang ihr aber mit der Wahl zum Sächsischen Landtag 2004. Als am Wahlabend die Stimmen ausgezählt waren, hatte die NPD 191.087 Stimmen (9,2 Prozent) erhalten und im Vergleich zu 1999 7,8 Prozentpunkte oder 161.494 Stimmen hinzugewonnen. Dies bedeutete zugleich, dass sie im neuen Landesparlament mit zwölf Abgeordneten vertreten sein würde. ${ }^{20}$

Die NPD konnte kein Direktmandat erringen. Die besten Erststimmenergebnisse erzielten Johannes Müller im Wahlkreis Sächsische Schweiz II mit 16,2, Klaus Baier in Annaberg mit 13,7 sowie Uwe Leichsenring im Wahlkreis Sächsische Schweiz I mit 11,8 Prozent. Das beste Zweitstimmenresultat konnten die Rechtsextremen im Wahlkreis Sächsische Schweiz II mit 15,1 Prozent verbuchen, gefolgt von Annaberg mit 14,0 und Riesa-Großenhain II mit 13,9 Prozent. ${ }^{21}$ Darüber hinaus gelang es ihnen, in fast der Hälfte (29 von 60) der sächsischen Wahlkreise zur drittstärksten Kraft aufzusteigen, und in jedem Wahlkreis konnte ein Zugewinn im Vergleich zur letzten Landtagswahl erreicht werden. ${ }^{22}$

\subsection{Wer wählte die NPD in Sachsen und warum?}

In den neuen Bundesländern tendieren viele Jungwähler zu einer rechtsextremen Partei, im Westen lässt sich eine derartige Korrelation nicht feststellen. ${ }^{23}$ In Sachsen votieren die 18bis 24-Jährigen mehr als doppelt so häufig für die NPD wie der Bevölkerungsdurchschnitt

17 Riesa-Großhain: 7,7 Prozent; Sächsische Schweiz: 7,0 Prozent; Muldentalkreis: 6,8 Prozent; Annaberg: 6,6 Prozent; Stollberg: 6,1 Prozent; Vogtlandkreis: 6,0 Prozent.

18 Kreistag Meißen-Radebeul: 5,1 Prozent (3 Sitze; 1999: 0 Sitze); Kreistag Muldentalkreis: 5,8 Prozent (3 Sitze; 1999: 0 Sitze); Kreistag Chemnitz-Land: 1,7 Prozent (1 Sitz; 1999: 0 Sitze); Kreistag Sächsische Schweiz: 9,1 Prozent (5 Sitze, 1999: 1 Sitz); Kreistag Landkreis Freiberg: 3,0 Prozent (1 Sitz; 1999: 0 Sitze).

19 Vgl. Homepage des NPD-Bundesverbandes, Kommunalwahl 2004, http://www.npd.de/npd_ info/wahlen/kommunalwahlen_2004.html [30. Juni 2005].

20 Vgl. Forschungsgruppe Wahlen e.V. (Hrsg.), Wahl in Sachsen. Eine Analyse der Landtagswahl vom 19. September 2004, Mannheim 2004, S. 7.

21 Vgl. Infratest dimap (Hrsg.), a.a.O., S. 39.

22 Vgl. Klaus Gertoberens, Die braune Gefahr in Sachsen. Personen, Fakten, Hintergründe, Dresden 2004, S. $16-54$.

23 Vgl. zur Situation in den 1990er Jahren Jürgen W. Falter, Wer wählt rechts? Die Wähler und Anhänger rechtsextremistischer Parteien im vereinten Deutschland, München 1994, S. 35 f. 
(21 zu 9,2 Prozent), damit ist die NPD unter den Jungwählern zweitstärkste Kraft hinter der CDU. Auch in den Altersgruppen der 25- bis 34-Jährigen, der 35- bis 44-Jährigen und der 45- bis 59-Jährigen kann die NPD Ergebnisse über dem Durchschnitt erzielen. Einzig die Senioren über 60 zeigen mit drei Prozent Zustimmung eine gewisse Immunität gegen eine rechtsextreme Wahlentscheidung. Ebenfalls bestätigt wird das eherne Wahlgesetz eines Männerüberschusses in der Wählerschaft extremistischer Parteien: Mit zwölf Prozent kann die NPD bei den Männern doppelt so gut wie bei den Wählerinnen abschneiden. ${ }^{24}$

Eine Differenzierung nach dem Bildungsgrad der NPD-Wählerschaft ergibt, dass die mittleren Bildungsschichten überdurchschnittlich vertreten sind, niedrige und hohe Abschlüsse dagegen sind seltener zu finden. Kombiniert man Bildung und Alter, so kann festgehalten werden, dass die Gruppe der jüngeren, formal niedrig gebildeten Menschen das größte Mobilisierungspotenzial der Partei darstellt. 26 Prozent der Wähler mit Hauptschulabschluss und 23 Prozent mit mittlerer Reife votierten für die NPD in Sachsen 2004. ${ }^{25}$

Soziodemographisch bestätigen sich die Befunde der Bildungsstruktur: Überdurchschnittlich sind Arbeiter (17 Prozent) und Arbeitslose (16 Prozent) vertreten, demgegenüber sind Rentner (vier Prozent), Angestellte (sechs Prozent) und Beamte (sechs Prozent) selten anzutreffen. Der Selbstständigenanteil bewegt sich im Landesdurchschnitt. ${ }^{26}$

Aus diesen Daten ergibt sich die folgende prozentuale Verteilung des NPD-Elektorats: 57 Prozent sind berufstätig, 20 Prozent arbeitslos und neun Prozent beziehen Rente; damit sind Arbeitslose mehr als doppelt so häufig in der NPD-Wählerschaft zu finden wie in der Bevölkerung. Die Rentner hingegen sind um das dreifache unterrepräsentiert. ${ }^{27}$

Hinsichtlich der politischen Herkunft der NPD-Wähler kann konstatiert werden, dass die Partei mit 70.000 Stimmen über die Hälfte ihrer Zugewinne aus dem bisherigen Nichtwählerlager mobilisieren konnte. Hinzu addiert werden noch 28.000 Erstwähler, die bei der letzten Landtagswahl nicht stimmberechtigt waren. Zudem konnte die NPD von allen anderen Parteien, mit Ausnahme der Grünen, Wähler abwerben. Den größten Gewinn verbucht sie von der CDU, insgesamt 39.000 Stimmen. Auch eine beträchtliche Anzahl (10.000) ehemaliger PDS-Wähler machte dieses Mal das Kreuz bei der NPD; von der SPD wechselten 3.000 Wähler zu den Rechtsextremen, von der FDP immerhin noch 1.000. Wahrscheinlich durch die Wahlabsprachen mit anderen rechten Parteien begünstigt, sind fast zehn Prozent der „sonstigen Parteien“ (10.000) in das Wählerlager der NPD gewandert. Aus dieser Bilanz ergibt sich ein Nettostimmenzugewinn von 160.000 Stimmen. ${ }^{28}$

Detaillierte Studien zu den Motiven von Wählern in Sachsen, sich für die NPD zu entscheiden, hat die Forschung bislang nicht erarbeitet. Fundierte Hypothesen, wie sie Klaus Liepelt für die NPD-Wählerschaft der sechziger Jahre entwickelt hat ${ }^{29}$, sind demnach nur schwer formulierbar; lediglich die Wahltagsbefragungen der Demoskopen liefern erste Hinweise. Danach sind überproportional viele NPD-Wähler mit den Leistungen der Bundesregierung unzufrieden. Sie sind der Meinung, dass sich die Koalition in Berlin zu wenig

24 Vgl. Infratest dimap (Hrsg.), a.a.O., S. 49 f.

25 Vgl. Forschungsgruppe Wahlen e.V. (Hrsg.), a.a.O., S. 52 f.

26 Vgl. Infratest dimap (Hrsg.), a.a.O., S. 51.

27 Vgl. Forschungsgruppe Wahlen e.V. (Hrsg.), a.a.O., S. 49.

28 Infratest dimap (Hrsg.), a.a.O., S. 35 f.

29 Vgl. Klaus Liepelt, Anhänger der neuen Rechtspartei. Ein Beitrag zur Diskussion über das Wählerreservoir der NPD, in: PVS, 8. Jg. (1967), S. $237-271$. 
um den Aufbau im Osten bemühe. ${ }^{30}$ Ebenso weisen 54 Prozent derer, die am 19. September 2004 für die NPD votierten, soziale Deprivationsgefühle auf. Sie glauben, dass sie weniger haben, als ihnen gerechterweise zustehe (Bevölkerung: 37 Prozent). ${ }^{31}$

Ebenfalls belegbar sind Züge eines rechtsextremistischen Orientierungsmusters. In der vorliegenden Datenquelle wurden zwar nicht alle Einzelstatements zur Bestimmung des latenten Rechtsextremismus abgefragt ${ }^{32}$; dennoch lassen verschiedene Antworten der Befragten auf eine überdurchschnittliche Verbreitung von rechtsextremistischen Einstellungen schließen. Nahezu die gesamte NPD-Anhängerschaft vertritt die Anschauung, dass es in Sachsen zu viele Ausländer gäbe. 96 Prozent sehen darin eine „Gefahr der Überfremdung “33, obwohl die derzeitige Ausländerquote in Sachsen gerade einmal 2,8 Prozent beträgt. ${ }^{34}$ Darüber hinaus halten 50 Prozent der NPD-Wähler die Demokratie für eine „nicht so gute Regierungsform" (Landesdurchschnitt 18 Prozent). ${ }^{35}$

Die Nationaldemokraten besitzen in den Augen der Wähler in der „Ausländerpolitik“ sowie in der „Bekämpfung der Kriminalität“ besondere Kompetenz. ${ }^{36}$ Interessant ist zudem, dass über die Hälfte der NPD-Anhänger (55 Prozent) ihre Wahlentscheidung nicht von der politischen Situation in Sachsen abhängig macht, sondern bundespolitische Themen in den Vordergrund stellt. ${ }^{37}$ Konkret nannten 57 Prozent der NPD-Wähler die Arbeitsmarktpolitik, insbesondere „Hartz IV“, als ausschlaggebendes Kriterium für ihre Wahl, für 38 Prozent war die Ausländerpolitik das entscheidende Motiv. ${ }^{38}$

\subsection{Wandel der Wählerschaft?}

Die NPD-Wähler in den 1960er Jahren, der erfolgreichsten Periode in der bisherigen Geschichte der NPD ${ }^{39}$, und die derzeitige Anhängerschaft weisen deutliche Abweichungen in ihrer Altersstruktur auf. Während damals besonders diejenigen Jahrgänge, die im Nationalsozialismus sozialisiert worden waren, zur Wahl der NPD tendierten, votierten 2004 in Sachsen überproportional viele Erst- und Jungwähler für die rechtsextreme Partei. Nach wie vor neigen nahezu doppelt so viele Männer zur Wahl der Partei wie Frauen.

Die formale Bildung der NPD-Wähler hingegen scheint gleich geblieben zu sein; in der Berufsstruktur ist allerdings ein deutlicher Unterschied auszumachen: Heute bildet die Ar-

30 Forschungsgruppe Wahlen e.V. (Hrsg.), a.a.O., S. 22 f.

31 Ebenda, S. 35.

32 Beispiel für eine Ermittlungsmethode zur Feststellung der rechtsextremen Orientierung innerhalb einer Bevölkerung: Jürgen W. Falter, a.a.O., S. 138. Falter unterteilt seine Rechtsextremismusskala in drei Teilbereiche: Nationalstolz und Kollektivdenken, Diktatur und Nationalsozialismus und Antisemitismus beziehungsweise Ausländerfeindlichkeit (ebenda, S. 137).

33 Vgl. Forschungsgruppe Wahlen e.V. (Hrsg.), a.a.O., S. 35 f.

34 Vgl. Toralf Staudt, Moderne Nazis, Die neue Rechte und der Aufstieg der NPD, Köln 2005, S. 213.

35 Vgl. Infratest dimap (Hrsg.), a.a.O., S. 95 f.

36 Ebenda, S. 76.

37 Vgl. Forschungsgruppe Wahlen e.V. (Hrsg.), a.a.O., S. 22.

38 Vgl. Viola Neu, Landtagswahl in Sachsen am 19.09.2004. Wahlanalyse (KAS-Onlinepublikationen), www.kas.de [1. Juli 2005]; Eckhard Jesse, a.a.O.

39 Zur Entwicklungsgeschichte der NPD: Uwe Hoffmann, Die NPD. Entwicklung, Ideologie und Struktur, Frankfurt am Main u.a. 1999. 
beiterschaft das größte Rekrutierungspotenzial, nicht mehr die Selbstständigen. Damaliges und heutiges Wählerreservoir haben gemeinsam, dass der Teil der Rentner äußerst gering ist.

Als Resümee ist demnach zu formulieren, dass die Veränderungen nicht so drastisch ausfallen, wie es gelegentlich in Öffentlichkeit, Presse oder Politikwissenschaft suggeriert wird. ${ }^{40}$ Die NPD-Wählerschaft hat sich im Laufe der Zeit verjüngt und proletarisiert, was als Folgewirkung der Neuausrichtung in der programmatischen Linie der Partei hin zu wirtschafts- und sozialpolitischen Schwerpunkten interpretiert werden kann.

\subsection{Der Wahlkampf der NPD}

Die NPD konzipierte ihren Wahlkampf in Sachsen von Beginn als Themen- und nicht als Personenwahlkampf. Bis auf ihren Spitzenkandidaten Holger Apfel waren keine Wahlplakate anderer Direktkandidaten sichtbar. ${ }^{41}$ Die Strategie war ganz auf die aktuelle politische Lage zugeschnitten. Für 87 Prozent der Sachsen stellte die Arbeitslosigkeit seinerzeit das größte Problem dar. ${ }^{42}$ Darüber hinaus zeigte sich eine deutliche Mehrheit der sächsischen Wähler mit der damaligen rot-grünen Bundesregierung und deren Ost-Politik unzufrieden. ${ }^{43}$ Infratest dimap ermittelte, dass für jeden Dritten (34 Prozent) bei der Stimmabgabe die Arbeitsmarktreformen eine entscheidende Rolle spielten. ${ }^{44}$ Diese Meinungslage instrumentalisierend setzte die NPD in ihrem Wahlkampf vordergründig auf Protest. Sie verband ihre Agitation allerdings stets mit fremdenfeindlichem Gedankengut. Dies verdeutlichen Wahlplakate mit Slogans wie "Grenze dicht für Lohndrücker“. ${ }^{45}$ Vordergründig spielten die ideologischen Aspekte im Wahlkampf jedoch eine untergeordnete Rolle. Die Partei präsentierte sich als Vertreterin des „kleinen Mannes“ und vermied es, ihre aggressiven programmatischen Ziele publik zu machen. ${ }^{46}$ Politiker- und Parteienkritik („Schnauze voll“) war ein weiteres zentrales Thema. ${ }^{47}$

Um den Erfolg abzusichern, betrieb die Partei neben einer wohl überlegten Themenwahl eine gut durchdachte Zielgruppenpolitik. Aus empirischen Studien war den Parteistrategen bekannt, dass die NPD hauptsächlich bei Erst- und Jungwählern überdurchschnittliche Resultate erwarten konnte. Aus diesem Grund versuchten sie, durch den Einsatz von neuen Medien die Jugendlichen für sich zu mobilisieren. Dem Vorbild der Neonaziszene folgend produzierte die NPD 25.000 CDs mit dem Titel „Schnauze voll? Wahltag ist Zahltag“, auf denen Musikstücke von rechtsextremen Skinheadbands und Liedermachern zu finden waren. Neben dem Einsatz neuer Medien verteilte die Partei auch eine Vielzahl „klassischer“

40 Vgl. beispielhaft: Bernd Neubacher, NPD, DVU-Liste D, Die Republikaner. Ein Vergleich ihrer Ziele, Organisationen und Wirkungsfelder, Köln 1996, S. 22.

41 Vgl. Franziska Brech, Ein halbes Jahr NPD im Sächsischen Landtag. Personen - Arbeitsstil - Entwicklungsperspektiven, Arbeitspapiere der Konrad-Adenauer-Stiftung Nr. 147/2005, S. 4.

42 Vgl. Forschungsgruppe Wahlen e.V. (Hrsg.), a.a.O., S. 32.

43 Vgl. ebenda, S. $22 \mathrm{f}$.

44 Vgl. Infratest dimap (Hrsg.), a.a.O., S. 45.

45 Vgl. Viola Neu, a.a.O.

46 Vgl. Die Welt vom 25. Januar 2005.

47 Vgl. Franziska Brech, a.a.O., S. 4. 
Wahlkampfmaterialien. Parteiangaben sprechen von 2,5 Millionen Wahlkampfzeitungen, zwei Millionen „Hartz-Flugblättern“, 500.000 Flugblättern mit landespolitischen Forderungen und 350.000 Landeswahlprogrammen. Zusätzlich wurden landesweit 35.000 Plakattafeln aufgehängt und mit Flugzeugen geworben, die mit NPD-Spruchbändern über das Land flogen. ${ }^{48}$

\section{Die gesellschaftliche Verwurzelung der NPD in Sachsen}

\subsection{In der „Mitte der Gesellschaft“ angekommen?}

Das gute Abschneiden der Nationaldemokraten in einigen Regionen des Freistaates kann für den aufmerksamen Beobachter keine Überraschung darstellen. Bereits in früheren Strategiepapieren und in Artikeln der „Deutschen Stimme“ hatte die Partei angekündigt, über ein kommunales Fundament Wahlerfolge vorzubereiten. Mit Blick auf Sachsen schrieb die Parteizeitung im Jahre 2000: „Um also die Voraussetzung für den Einzug in den Landtag in dreieinhalb Jahren zu schaffen, müssen weitere Lokalgrößen und weitere regional bekannte Politiker der NPD aufgebaut werden. “49

Dass mit diesem Konzept Erfolge zu verbuchen sind, zeigt eine Analyse der Wahlergebnisse. In bestimmten Gebieten kann die NPD seit geraumer Zeit mit achtbaren Ergebnissen aufwarten. In Rheinhardtsdorf-Schöna hatte sich das sachsenweit beste Ergebnis bei den Kommunalwahlen 2004 mit 25,2 Prozent der Stimmen bereits bei den Bundestagswahlen 1998 und 2002 angedeutet. 1998 votierten 6,9 Prozent der Wähler für die Partei, vier Jahre später sogar 9,4 Prozent. ${ }^{50}$ In Königstein bilden die Nationaldemokraten schon seit 1999 die drittstärkste Fraktion im Stadtrat. Nachdem sie 1994 nicht zur Wahl angetreten waren, erzielten sie 1999 aus dem Stand 11,8 Prozent der Wählerstimmen. ${ }^{51}$ Beide Orte gehören zum Landkreis Sächsische Schweiz, dessen NPD-Kreisverband mit dem Arzt Johannes Müller (Vorsitzender) und dem mittlerweile verstorbenen Uwe Leichsenring zwei NPD-Landtagsabgeordnete vorstehen beziehungsweise vorstanden. Ein Blick auf die übrigen Kreistagsmitglieder zeigt ferner, dass mit Michael Jacobi (Klempnermeister), Barbara Müller (Krankenschwester) und Steffen Richter (Unternehmer) weitere Personen des kleinbürgerlichen Milieus die Partei vor Ort repräsentieren. ${ }^{52}$ Diese These wird durch eine weiterführende Untersuchung des gesamten Kreisvorstandes bestätigt; es fällt auf, dass nicht der „gesellschaftliche Außenseiter“, sondern der vor Ort anerkannte Bürger die lokale Führungsebene der Partei dominiert. ${ }^{53}$

Darüber hinaus versucht die NPD zunehmend, im vorpolitischen Raum Fuß zu fassen. Vertreter von zivilgesellschaftlichen Initiativen berichten, dass Parteifunktionäre seit Jahren durch ihr sozialpolitisches Engagement auf sich aufmerksam machen. Sie seien Mitglieder

48 Vgl. Deutsche Stimme, Nr. 10/2004.

49 Deutsche Stimme, Nr. 04/2000.

50 Vgl. Klaus Gertoberens, a.a.O., S. 58.

51 Vgl. ebenda, S. $59 \mathrm{f}$.

52 Vgl. Homepage des NPD-Kreisverbandes Sächsische Schweiz, www.npd-saechsische-schweiz.de [8. Juli 2005].

53 Vgl. ebenda. 
in den Feuerwehren, im Fußballverein oder helfen bei der Organisation von Kirchenveranstaltungen. Zudem betreiben sie Kneipen, organisieren Konzerte für die Jugend oder richten politische Schulungen aus. ${ }^{54}$ So gelingt es, insbesondere die lokale Jugendszene an sich zu binden. Während aus Geldmangel die offiziellen Freizeitangebote gekürzt werden, investiert die NPD gerade hier. Durch Konzerte mit rechtsextremen Skinheadbands oder Zeltlager schafft sie es, nicht nur denjenigen Teil der Jugend an sich zu binden, der ohnehin über eine rechtsextreme Orientierung verfügt, sondern sie politisiert die bis dahin eher unpolitisch eingestellten Jugendlichen in ihrem Sinne. ${ }^{55}$ Die NPD stößt damit in eine soziokulturelle Lücke, die vielfach von den staatlichen Stellen nicht ausgefüllt wird. Die Partei fördert ganz gezielt die rechtsextreme Jugendszene in den neuen Bundesländern, die sich dort in den letzten 15 Jahren deutlich vergrößern konnte. ${ }^{56}$

Die NPD gibt sich örtlich das Image der „sozialen Wohltäterin“. Junge Parteimitglieder besuchen Altersheime oder helfen Familien bei ihren Einkäufen; NPD-Ortsverbände rufen Bürgerinitiativen ins Leben, die sich für bessere Lebensqualität oder gegen Ausländer einsetzen. Die soziale Akzeptanz führt mittlerweile sogar so weit, dass mancherorts die Parteigliederungen zu „runden Tischen gegen Gewalt" eingeladen werden. ${ }^{57}$

Durch die Integration von vorrangig aktuellen und lokalpolitischen Themen in die politische Agenda der Partei steigert die NPD ihre soziale Akzeptanz in den örtlichen Gesellschaften. ${ }^{58}$ Dabei zeichnet sich deren Kommunalpolitik nicht zuerst durch rechtsextreme Rhetorik, sondern durch eine pragmatische und problemorientierte Politik aus. ${ }^{59}$ Die Parteifunktionäre werden daher von der Bevölkerung nicht als „Rechtsextremisten“ wahrgenommen, es dominiert vielmehr das Bild des „normalen Bürgers“ als Teil der örtlichen Gemeinschaft. Kontakte der Nationaldemokraten zu neonazistischen Kreisen werden durch die Bürger ignoriert; es stört nicht, dass Uwe Leichsenring Verbindungen zu den Neo-Nazis der Skinheadkameradschaft "Skinheads Sächsische Schweiz“ (SSS) nachgewiesen worden sind $^{60}$ oder dass bei Michael Jacobi im Zuge der Ermittlungen gegen die SSS ein Sprengstoffarsenal in der heimischen Garage gefunden wurde. ${ }^{61}$ Die Akzeptanz der NPD ist indes derart weit vorgeschritten, dass sogar Kommunalpolitiker wie der Bürgermeister von Rheinhardtsdorf-Schöna Arno Suddar (CDU) den Nationaldemokraten bescheinigt, dass man mit diesen besser zusammenarbeiten könnte als mit der PDS. ${ }^{62}$

Was sich auf der lokalen politischen Bühne andeutet, findet die Vollendung im Landesparlament: Bei den Wahlen zum Ministerpräsident und zur Ausländerbeauftragten erhielten die NPD-Kandidaten jeweils zwei Stimmen mehr, als ihre Fraktion Sitze im Parlament hat; bei der Bestimmung der Mitglieder des Landesjugendhilfeausschusses unterstützten fünf Nicht-NPD-Mitglieder deren Wahlvorschlag durch ihre Stimme. ${ }^{63}$ Nach den Ein-

54 Zitiert nach: http://www.tolerantes-sachsen.de/news_enzeln.php4?ID=196 [1. Oktober 2004].

55 Vgl. Der Spiegel, Nr. 02/2001.

56 Vgl. Toralf Staudt, a.a.O., S. 14.

57 SPD-Bundestagsfraktion, Rechtsextremismus in Deutschland. Eine Handreichung, Berlin 2005, http://www.spdfraktion.de/rs_datei/0,,5071,00.pdf [12. Mai 2005].

58 Vgl. Der Tagesspiegel vom 7. Januar 2005.

59 Vgl. Toralf Staudt, a.a.O., S. 134.

60 Vgl. Franziska Brech, a.a.O., S. 10.

61 Vgl. Der Spiegel, Nr. 40/2004.

62 Zitiert nach: Frankfurter Rundschau Online [28. August 2004].

63 Homepage des Stern, Bieder, brav und brandgefährlich [2. Februar 2005]. 
schätzungen des sächsischen Verfassungsschutzes scheint die NPD in der „Mitte der sächsischen Gesellschaft“ angekommen zu sein - zumindest in ihren Hochburgen. Die Parteifunktionäre haben Kontakt zu den Menschen und genießen deren Sympathie. ${ }^{64}$

\subsection{Die sächsische NPD-Landtagsfraktion}

Auffällig ist, dass nur sieben von zwölf der sächsischen Landtagsmitglieder ${ }^{65}$ auch tatsächlich in Sachsen geboren sind. ${ }^{66}$ Den Fraktionsvorsitz hat mit dem aus Hildesheim stammenden Holger Apfel ein Westdeutscher übernommen. Mit Müller und Leichsenring, der auch als Parlamentarischer Geschäftsführer der Landtagsfraktion fungiert(e), sind jedoch zwei Sachsen als die beiden Stellvertreter von Apfel in den weiteren exponierten Positionen der Fraktion vertreten. Mit Gitta Schüßler gehört der Fraktion nur eine Frau an, wodurch sich eindeutig die unterdurchschnittliche Repräsentanz des weiblichen Geschlechts in Mitgliedschaft und Elektorat der NPD widerspiegelt.

Die NPD-Fraktion bildet soziodemographisch keine homogene Gruppe; es sind sowohl akademisch gebildete Mitglieder, Repräsentanten mit handwerklicher oder kaufmännischer Ausbildung als auch Vertreter mit einer ausschließlichen Parteikarriere zu finden. Immerhin ein Drittel arbeitete vor der Tätigkeit als Landtagsabgeordnete hauptamtlich bei der Partei oder bei der „Deutschen Stimme“ in Riesa. ${ }^{67}$ Einen Hochschulabschluss können Müller und Gansel vorweisen, daneben verfügen mit Apfel und Leichsenring nur zwei weitere Fraktionsmitglieder über einen hohen Bildungsabschluss. Eine kaufmännische Ausbildung haben vier Fraktionsmitglieder abgeschlossen ${ }^{68}$, aus dem Handwerksgewerbe stammen fünf Personen. ${ }^{69}$ Selbstständig als Unternehmer waren sechs Nationaldemokraten. ${ }^{70}$ Im Gegensatz zu den Abgeordneten der anderen Parteien bekleidete in der NPD-Fraktion nur Uwe Leichsenring ehrenamtliche Positionen in Aufsichts-, Betriebs- oder Verwaltungsräten. Dies könnte darauf hindeuten, dass die NPD in der lokalen Wirtschaft, aber auch in der gemeinnützigen Struktur der Kommunen noch nicht derart verwurzelt ist, wie dies der erste Blick suggeriert. Auffallend ist ferner, dass alle Angehörigen der sächsischen NPD-Landtagsfraktion innerhalb der Partei führende Funktionen innehaben. Dies beginnt mit der

64 Vgl. O. V., Feste Strukturen. Rechtsextremisten sind mancherorts in der Mitte der Gesellschaft verankert, in: Klaus Gertoberens, a.a.O., S. 88 f., S. 89.

65 Alle Angaben zu den NPD-Abgeordneten stammen aus: Andreas Holzapfel, Sächsischer Landtag. 4. Wahlperiode. Volkshandbuch, Rheinbreitbach 2004 sowie NPD-Fraktion im Sächsischen Landtag (Hrsg.), 9,2 \% für Sachsen. Wir danken unseren Wählerinnen und Wählern, Dresden 2004. Die Analyse erfolgt für diejenigen zwölf NPD-Mitglieder, die ihr Mandat im September 2004 angetreten haben, spätere Veränderungen innerhalb der Fraktion werden zu einem nachfolgenden Zeitpunkt behandelt.

66 Uwe Leichsenring, Johannes Müller, Matthias Paul, Gitta Schüßler, Mirko Schmidt, Jürgen Schön, Klaus Baier.

67 Holger Apfel, Alexander Delle, Jürgen Gansel (Deutsche Stimme); als hauptamtlicher Angestellter der NPD: Jürgen Schön.

68 Holger Apfel, Alexander Delle, Gitta Schüßler, Winfried Petzold.

69 Uwe Leichsenring, Matthias Paul, Mirko Schmidt, Jürgen Schön, Klaus Baier.

70 Uwe Leichsenring, Matthias Paul, Gitta Schüßler, Winfried Petzold, Klaus Baier und Klaus-Jürgen Menzel. 
Geschäftsführung eines Kreisverbandes über den sächsischen NPD-Vorsitzenden Winfried Petzold bis hin zum stellvertretenden Bundesvorsitzenden Apfel.

Ähnlich wie in der Landtagsfraktion selbst sind auch innerhalb des Mitarbeiterstabes der Fraktion zahlreiche rechtsextreme Prominente anzutreffen. Mit der Verpflichtung von Karl Richter als „Leiter des Parlamentarischen Beratungsdienstes der NPD-Fraktion“ konnte einer der „führenden rechtsextremen Publizisten und intellektuellen Köpfe“ in die Fraktionsarbeit integriert werden. Bekannt wurde Richter durch seine Tätigkeit als Chefredakteur der Zeitschrift „Nation\&Europa“ (1991 bis 1997) ${ }^{71}$; zwischen 1989 und 1994 arbeitete er zeitweise als Referent für den Abgeordneten der Republikaner (REP) im Europäischen Parlament, Harald Neubauer. Richter versucht, die sächsische Fraktion als „Denkfabrik der Partei“ zu etablieren. ${ }^{72}$ Neben ihm gehören sechs weitere Personen dem Beratungsdienst der Fraktion an. Besonders erwähnenswert sind die beiden letzten Vorsitzenden der JN, Sascha Rossmüller (1999 bis 2002) und Stefan Rochnow (JN-Bundesvorsitzender seit 2002) ${ }^{73}$, die beide zu einer neuen Generation der NPD-Funktionäre zu zählen sind, die sich vollkommen der Parteiarbeit verschrieben haben. ${ }^{74}$ Die Pressestelle der Fraktion hat der Sachse Holger Szymanski übernommen, der auch die Pressearbeit des NB Dresden bestreitet. ${ }^{75}$ Neben Szymanski ist Kerstin Lorenz ${ }^{76}$ für die weitere Öffentlichkeitsarbeit zuständig. Bis 2004 fungierte sie als Landesvorsitzende der REP, zog vor der Landtagswahl 2004 deren Kandidatur eigenmächtig zurück und steigerte so die Erfolgschancen der NPD, der sie medienwirksam einen Tag vor dem Urnengang beitrat. Zuvor hatte sie bereits im NB eng mit anderen nationaldemokratischen Funktionären zusammengearbeitet. ${ }^{77}$ Die Geschäftsführung der Fraktion hat der Multifunktionär Peter Marx übernommen ${ }^{78}$, der gleichzeitig noch den Landesvorsitz in Rheinland-Pfalz innehat und stellvertretender Bundesvorsitzender ist. Die für die parlamentarische Arbeit nötige Erfahrung konnte Marx als Fraktionsgeschäftsführer der NPD-Fraktionen in der Frankfurter Stadtverordnetenversammlung und im Landkreis Darmstadt erlangen. ${ }^{79}$

Bilanzierend lässt sich konstatieren, dass die Landtagsfraktion der NPD eine ähnliche Struktur aufweist wie die lokale Parteiorganisation. Das von den Medien gezeichnete Bild des „Rechtsextremisten in Springerstiefeln und Bomberjacke“ sucht man in der Fraktion vergebens, vielmehr verfügen alle Mitglieder über eine bürgerliche Existenz. Die NPD-Parlamentarier erwecken einen seriösen Eindruck, die Presse verwendet gelegentlich die Attri-

71 Zitiert nach: Blick nach Rechts, Ausgabe 23/2004 - Job-Maschine, www.bnr.de [18. Mai 2005].

72 Vgl. Franziska Brech, a.a.O., S. 16.

73 Tätigkeit bis September 2006, danach Wechsel zur Landtagsfraktion in Mecklenburg-Vorpommern.

74 Vgl. Franziska Brech, a.a.O., S. 17 f.

75 Vgl. Gefragter Mitarbeiter, in: Blick nach Rechts, Ausgabe 22/2004, www.bnr.de [18. Mai 2005]. Szymanski ist darüber hinaus zum stellvertretenden Fraktionsgeschäftsführer aufgestiegen.

76 Lorenz ist am 5. September 2005 verstorben, vgl. Klartext Nr. 05/2005. Informationen der NPDFraktion im Sächsischen Landtag, S. 2.

77 Vgl. Franziska Brech, a.a.O., S. 18.

78 Die Geschäftsführung hat mittlerweile Frank Ahrens übernommen, da Peter Marx nach dem Erfolg bei der Landtagswahl in Mecklenburg-Vorpommern nach Schwerin gewechselt ist, um hier in selbiger Position die Geschicke der Fraktion zu lenken.

79 Vgl. Franziska Brech, a.a.O., S. 14 f. 
bute „bieder und brav“ zur Beschreibung. ${ }^{80}$ Auch soziodemographisch stellt sich die Partei wie ein verkleinerter Querschnitt der Bevölkerung dar; von Akademikern über Handwerker bis hin zum Landwirt sind viele Berufszweige vertreten. Den im Vergleich zu den übrigen Landtagsfraktionen unterdurchschnittlichen Bildungsgrad versucht die Partei durch die Professionalisierung ihres Mitarbeiterstabes zu kompensieren.

\subsection{Die öffentliche Bewertung der sächsischen NPD}

Das Verhältnis der sächsischen Öffentlichkeit zur NPD ist einerseits von Akzeptanz und Integration der Partei in die lokale Gesellschaft, andererseits durch eine massive Ablehnung gekennzeichnet.

Die NPD ist besonders in jenen Regionen erfolgreich, in denen sie von Bürgern des kleinbürgerlichen Milieus vertreten wird, die sich in der lokalen Gesellschaft eine gewisse Anerkennung erarbeitet haben. Dies ist ebenso in der landesweiten Wahrnehmung der NPD als Partei nachvollziehbar. So stellte Infratest dimap fest, dass fast ein Viertel der Bevölkerung die NPD als eine demokratische Partei wie jede andere einstuft. ${ }^{81}$ Eckhard Jesse führt dies auf den für den Durchschnittsbürger nicht nachvollziehbaren Ausgang des Verbotsverfahrens zurück. ${ }^{82}$ Gleichzeitig befürchteten allerdings 72 Prozent der sächsischen Bürger negative Folgen für ihr Bundesland, falls die NPD tatsächlich in den Landtag in Dresden einziehen könnte. Aber immerhin zwölf Prozent bekundeten vor der Wahl, dass sie das Überschreiten der Fünfprozenthürde begrüßen würden. ${ }^{83}$

\section{Protest- oder Bekenntniswahl?}

Wenige Tage nach der Landtagswahl in Sachsen im September 2004 war sich die Öffentlichkeit einig, dass das gute Abschneiden der NPD mit dem Protestverhalten der Wähler zu begründen sei. Unterstützung erhielt diese Interpretation aus der Wahlforschung ${ }^{84}$ und der Politikwissenschaft. ${ }^{85}$ In der Tat spricht zunächst eine Vielzahl von Argumenten für die Hypothese einer rationalen Protestwahl. Mit „Hartz IV“ bestimmte ein bundespolitisches Thema die Agenda und gab für einen Großteil der nationaldemokratischen Wähler den Ausschlag für ihre Wahlentscheidung. Viele Menschen in der ehemaligen DDR sehen sich als Verlierer der Einheit, und meinen, dass sie nicht das besitzen, was ihnen gerechterweise zustehe. Viola Neu verwendet in diesem Zusammenhang den gängigen Begriff der „Modernisierungsverlierer" 86 .

80 Homepage des Stern, Bieder, brav und brandgefährlich [2. Februar 2005].

81 Vgl. Infratest dimap (Hrsg.), a.a.O., S. 86.

82 Vgl. taz vom 30. Oktober 2004, S. 6.

83 Vgl. Forschungsgruppe Wahlen e.V. (Hrsg.), a.a.O., S. 26.

84 Vgl. ebenda, S. 12.

85 Vgl. Viola Neu, a.a.O.; Eckhard Jesse, a.a.O.

86 Viola Neu, a.a.O. 
Innerhalb des NPD-Elektorats spricht allerdings fast ein Fünftel (18 Prozent) davon, die Partei aus ideologischer Überzeugung gewählt zu haben. ${ }^{87}$ Dies korrespondiert mit den Untersuchungen von Infratest dimap. Das Berliner Meinungsforschungsinstitut rechnet elf Prozent der NPD-Anhänger eine langfristige Parteibindung zu. ${ }^{88}$ Für diese Wähler darf demnach der Begriff „Stammwähler“ verwendet werden. Richard Stöss schätzt sogar, dass die Hälfte der NPD-Wähler genau weiß, was sie wählt. Diese hätten nicht nur ein Problem mit „Hartz IV“, sondern lehnten vielmehr das System Bundesrepublik ab. ${ }^{89}$ Die Funktionäre der Partei selbst beziffern ihr Stammpotenzial im Freistaat auf fünf Prozent. ${ }^{90}$ Weiterhin geben 73 Prozent derjenigen, die bei den Wahlen für die NPD votiert haben, an, dies wegen deren Lösungsvorschlägen zu Sachfragen getan zu haben ${ }^{91}$ - ebenfalls ein Indiz für die Identifikation mit der rechtsextremen Partei. Diese Wähler haben sich bewusst für diese Partei entschieden - nicht trotz, sondern wegen ihrer Ideologie. Von den Vertretern der "Protestwahlhypothese“ wurde zudem die lokale Verwurzelung der Partei in bestimmten Gebieten Sachsens nur unzureichend berücksichtigt. Die Hypothese von den „Einheitsverlierern" ist demnach nicht so einfach auf die Hochburgen der NPD zu übertragen; diese verfügen im Vergleich zu den übrigen sächsischen Landkreisen über eine überdurchschnittlich gute wirtschaftliche Situation. ${ }^{92}$ Die Parteimitglieder und Funktionäre sind hier anerkannt, akzeptiert und in die örtliche Gesellschaft integriert. ${ }^{93}$ Dies ermöglicht eine Identifikation der Bürger mit der Partei. Obwohl sie nicht als dezidiert rechtsextreme Partei wahrgenommen wird, wird ihr rechtsextremistisches Gedankengut, gleichsam durch die Hintertür, salonfähig gemacht. Die NPD kann überall dort ihre größten Erfolge feiern, wo sie von Bürgern aus dem kleinbürgerlichen Milieu vertreten wird, die neben Seriosität auch eine gewisse Kompetenz ausstrahlen. Ihr ist es so gelungen, bislang weitgehend unbeobachtet, in der kommunalen Arbeit Strukturen aufzubauen, die ihren Erfolg nun begünstigen.

Stabile Parteistrukturen fördern also regionale Wahlerfolge. Sie üben gleichzeitig eine gewisse Attraktivität auf die Bevölkerung aus, wie die gestiegenen Mitgliederzahlen des Landesverbandes zeigen. Um langfristigen Erfolg beim Wähler zu erreichen, ist gefestigte Organisation in regionalen Schwerpunktzentren - gerade für eine Splitterpartei wie die NPD - unabdingbare Voraussetzung.

Bereits 1994 hatte Jürgen Falter im Rahmen seiner Theorie zur Erklärung rechtsextremer Wahlerfolge eingeräumt, dass bei einem extrem hohen Anteil an Rechtswählern ein größerer Anteil der Stimmen von „reinen Protestwählern“ stammen könnte. ${ }^{94}$ Dies wurde bei der sächsischen Landtagswahl bestätigt. Zwar verfügt der Freistaat über eine rechtsextreme Stammwählerschaft der NPD, diese scheint jedoch nicht allein in der Lage, der Partei den Sprung über die Fünfprozenthürde zu ermöglichen. Addiert man zu dem Reservoir die reinen Protestwähler hinzu, kann das gute Abschneiden der NPD erklärt werden. Um den lokalen Besonderheiten in Sachsen Rechnung zu tragen, ist es also nötig, die NPD-Anhän-

87 Vgl. Forschungsgruppe Wahlen e.V. (Hrsg.), a.a.O., S. 26.

88 Vgl. Infratest dimap (Hrsg.), a.a.O., S. 47.

89 Vgl. Jörg Albinsky, Der lange Marsch der NPD. Politische Versäumnisse rächen sich bitter, in: Klaus Gertoberens, a.a.O., S. 11 ff., S. 12.

90 Gespräch mit Jürgen Gansel, MdL, am 22. Juni 2005.

91 Vgl. Infratest dimap (Hrsg.), a.a.O., S. 47.

92 Vgl. Forschungsgruppe Wahlen e.V. (Hrsg.), a.a.O., S. 19 f.

93 Vgl. auch die Fernsehreportage „Lauter nette Leute“ auf Phoenix (5. März 2005).

94 Vgl. Jürgen W. Falter, a.a.O., S. 152. 
ger zu differenzieren. Auf der einen Seite hat sich landesweit eine rechtsextreme Stammwählerschaft der NPD herauskristallisiert; zusätzlich haben sich eine Reihe „reiner Protestwähler" dazu entschlossen, für die Partei zu stimmen. Mit Blick auf die Theorien der Wahlforschung kann dementsprechend ein „sowohl als auch“ von rationaler Protest- und ideologischer Bekenntniswahl für die sächsische Landtagswahl 2004 konstatiert werden.

\section{Die sächsische NPD seit 2005}

Während 2004 durchaus als das erfolgreichste Jahr in der Geschichte der NPD seit den sechziger Jahren gelten kann, scheint bereits ein Jahr später wieder Ernüchterung in der Partei eingetreten zu sein. Selbst der ansonsten erfolgsverwöhnte Landesverband Sachsen bildet keine Ausnahme; im Gegenteil, die Probleme und innerparteilichen Differenzen, die in anderen Verbänden ebenso wie auf Bundesebene beobachtet werden können, treten im Freistaat mit weitreichenden Folgen für die Partei zu Tage.

Zunächst ist festzuhalten, dass die NPD bei der Bundestagswahl 2005 ihre hochgesteckten Ziele nicht verwirklichen konnte. Die Strategie, durch den Gewinn von drei Direktmandaten in ihrem Stammland Sachsen den Einzug in den Bundestag in Fraktionsstärke zu sichern, scheiterte kläglich. In keinem einzigen Wahlkreis konnten ihre Kandidaten den demokratischen Parteien nur annähernd gefährlich werden. ${ }^{95}$ Allerdings erzielte sie mit 4,8 Prozent der in Sachsen abgegebenen Stimmen ein für ihre Verhältnisse sehr gutes Ergebnis bei einer Bundestagswahl. Das Resultat von 2002 (1,4 Prozent) konnte gut verdreifacht werden, stellt aber fast eine Halbierung des Spitzenergebnisses bei der Landtagswahl vor Jahresfrist dar.

Die Fraktion im Dresdner Landtag beschäftigten darüber hinaus Probleme ganz anderer Art: Nachdem zunächst der Abgeordnete Mirko Schmidt kurz vor Weihnachten 2005 seinen Austritt aus Fraktion und Partei bekannt gegeben hatte ${ }^{96}$, folgten innerhalb weniger Tage die Parlamentarier Klaus Baier und Helmut Schön diesem Beispiel. ${ }^{97}$ Schmidt und Baier begründeten ihren Austritt mit menschlicher und politischer Enttäuschung über die Entwicklung der Fraktion. ${ }^{98}$ Ferner kritisierten sie den autoritären Führungsstil innerhalb der Partei und deren zunehmende ideologische Ausrichtung am historischen Nationalsozialismus. ${ }^{99}$ Verantwortlich für diesen Kurs seien insbesondere die nach Sachsen „zugereisten NPD-Funktionäre“. Gemeinsam ist allen drei Abtrünnigen die Kritik an einer „West-Dominanz " in der Fraktion. ${ }^{100}$ Ebenso blieb die tägliche politische Arbeit im Parlament nicht von Angriffen der Ausgetretenen verschont: Während „Anti-Amerikanismus“ und historische Themen die Debatten der Fraktion dominieren würden, sei die Auseinandersetzung

95 Vgl. Eckhard Jesse, Das Auf und Ab der NPD, in: APuZ, B 42 (2005), S. 31 - 38, S. 38.

96 Vgl. Verfassungsschutz half Abgeordneten bei Ausstieg, auf: Spiegel Online [7. März 2006].

97 Vgl. Dritter Abgeordneter verlässt sächsische NPD-Fraktion, auf: Spiegel Online [7. März 2006].

98 Vgl. Wie sich die NPD selbst zerlegt, auf: Spiegel Online [7. März 2006].

99 Vgl. Dritter Abgeordneter verlässt sächsische NPD-Fraktion, auf: Spiegel Online [7. März 2006].

100 Vgl. Hauen und Stechen, in: Blick nach Rechts, Ausgabe 01/2006, www.bnr.de, S. 2 f., S. 3. Schön kritisierte die Westdominanz, aber wohl nicht die ideologische Ausrichtung von Partei und Fraktion. 
mit der "Sozialen Frage“ zunehmend in den Hintergrund getreten. Aber gerade wegen ihrer Sozialpolitik sei die Partei im September 2004 gewählt worden; somit vertrete sie nicht mehr die Interessen des größten Teils ihrer Wähler, argumentieren Schön, Schmidt und Baier. ${ }^{101}$

Erwartungsgemäß reagierte die Parteiführung auf die Austrittswelle, indem sie ihre ehemaligen Kollegen scharf attackierte: Für die Fraktion halte sich der Schaden in Grenzen, da es sich bei den drei ehemaligen NPD-Abgeordneten allesamt um „Hinterbänkler“ im Parlament gehandelt habe. ${ }^{102}$ Offensichtlich ist das Gegenteil der Fall: Schön hatte nicht unerheblich dazu beigetragen, die NPD nach der Wiedervereinigung in Sachsen aufzubauen, stand in den ersten zwei Jahren dem Landesverband als Vorsitzender vor (1990 bis 1992). Bis zuletzt fungierte er darüber hinaus als sein Schatzmeister, seit 1995 außerdem als stellvertretender Landesvorsitzender. ${ }^{103}$ Auf Bundesebene war er ebenfalls aktiv - von 1996 bis 2004 amtierte er als stellvertretender Bundesvorsitzender. Schmerzlich bemerkbar machen dürfte sich der Verlust von Schön noch in einem weiteren Sinne: Seit 1995 war er hauptamtlicher Angestellter der Rechtsextremen. ${ }^{104}$ Mit Schmidt und Baier verliert die Partei zwei wichtige Vertreter der neuen Generation von Rechtsextremen mit einem um Seriosität bemühten Image. Ebenso wie Schön waren sie Mitglied des Landesvorstandes, Schmidt wurde auf dem letzten ordentlichen Parteitag sogar zu einem der Vertreter des Vorsitzenden Winfried Petzold gewählt. Zu seinen zahlreichen Parteiämtern zählten der Landesvorsitz der Kommunalpolitischen Vereinigung der NPD und zeitweise auch deren Stellvertreterposten auf Bundesebene. Seit 1999 ist er Stadtrat in Meißen, seit der Kommunalwahl 2004 verfügt er außerdem über einen Sitz im Kreistag. Baier ist ebenfalls Stadtrat, seit 2004 vertrat er die NPD im Kommunalparlament von Annaberg-Buchholz und ist dort nun parteiloser Mandatsträger. ${ }^{105}$ Darüber hinaus konnte er bei der Landtagswahl überregional auf sich aufmerksam machen. Sowohl bei den Erst- als auch bei den Zweitstimmen erzielte er das landesweit zweitbeste Ergebnis der NPD, übertroffen nur von Johannes Müller im Wahlkreis Sächsische Schweiz II. ${ }^{106}$ Damit war Baier sogar erfolgreicher als der vielen NPD-Mitgliedern als neuer Hoffnungsträger geltende Leichsenring im Wahlkreis Sächsische Schweiz I. Schmidt, Baier und Schön gehören dem Sächsischen Landtag nun als fraktionslose Abgeordnete an.

Aus der Tatsache, dass beim Ausstieg von Schmidt der sächsische Verfassungsschutz Hilfestellung gab und diesen sogar ins offizielle Aussteigerprogramm aufgenommen habe ${ }^{107}$, konstruierten die Rechtsextremen in gewohnter Weise eine Verschwörungstheorie ${ }^{108}$ : Es habe bereits vor den Austritten in der NPD Mutmaßungen gegeben, dass es sich bei Schmidt und Baier um so genannte V-Männer des sächsischen Landesamtes für Verfassungsschutz gehandelt habe. ${ }^{109}$ Die Behörde selbst bestritt indes die Vorwürfe.

$101 \mathrm{Vgl}$. Wie sich die NPD selbst zerlegt, auf: Spiegel Online [7. März 2006].

102 Vgl. Reisende soll man nicht aufhalten, in: Klartext Nr. 06/2005. Informationen der NPD-Fraktion im Sächsischen Landtag, S. 3.

103 Vgl. Hauen und Stechen, in: Blick nach Rechts, a.a.O., S. 3.

104 Vgl. NPD-Fraktion im Sächsischen Landtag (Hrsg.), a.a.O.

105 Vgl. ebenda.

106 Vgl. Tabelle der Wahlergebnisse bei Franziska Brech, a.a.O., S. 7 f.

107 Vgl. Verfassungsschutz half Abgeordneten bei Ausstieg, auf: Spiegel Online [7. März 2006].

108 Vgl. Reisende soll man nicht aufhalten, in: Klartext Nr. 06/2005, a.a.O., S. 3.

109 Vgl. Wie sich die NPD selbst zerlegt, auf: Spiegel Online [7. März 2006]. 
Entgegen den offiziellen Behauptungen stellt der Verlust der drei Abgeordneten also durchaus eine Belastungsprobe für die NPD dar. Neben innerparteilichen Konfrontationen, in deren Verlauf der „organisatorische Notstand“ über vier Kreisverbände (Löbau-Zittau, Görlitz, Annaberg-Buchholz, Meißen) verhängt wurde, versuchte die Parteiführung, bei den verbliebenen Verbänden Unterstützungsbekundungen für ihr Vorgehen einzuholen, was zu einer Spaltung des sächsischen Landesverbandes führen könnte. ${ }^{110}$ Zusätzlich hat die Partei, den Angaben von Schön zufolge, 110 Mitglieder verloren, die sich mit den Ausgetretenen solidarisierten. ${ }^{111}$ Parteiangaben sprechen hingegen von „nur“ 23 Austritten zwischen Dezember 2005 und Februar 2006. Den Abgängen stünde jedoch eine fast dreimal so hohe Anzahl von Neumitgliedern (63) gegenüber. ${ }^{112}$ Probleme dürften auch die finanziellen Einbußen durch den Verlust von drei Fraktionsmitgliedern bereiten. Der Verband muss allerdings nicht nur auf 85.680 Euro im Jahr verzichten, sondern verliert ferner einen Sitz in den Ausschüssen des Landtages. Sie ist nun nicht mehr mit zwei, sondern nur noch mit einem Mitglied in den Gremien vertreten. ${ }^{113}$

Nach wenigen Monaten scheinbarer Ruhe setzte die NPD im November und Dezember 2006 die Selbstdezimierung fort: Zunächst schloss die Fraktion den Abgeordneten KlausJürgen Menzel aus; wenig später folgte gar sein kompletter Rückzug aus der Partei. Offiziell begründet wurde die Entscheidung des Fraktionsvorstandes mit dem Finanzgebaren des Parlamentariers, der mehrmals Darlehen verschiedener Höhe bei Bürgern aufgenommen, diese allerdings nicht zurückgezahlt habe. ${ }^{114}$ Weit wichtiger dürfte für die Partei allerdings die politische Dimension des Vorgangs gewesen sein: Menzel hatte sich in einem Interview zum „Führer“ bekannt und somit dem selbst gewählten Image der NPD schweren Schaden zugefügt. ${ }^{115}$ In der Folgezeit setzte er, der seither als fraktionsloser Abgeordneter dem Landtag angehört, seine Provokationen fort: Er schmuggelte eine scharfe Pistole in das Landtagsgebäude oder legte in einer Debatte einige Patronen auf das Rednerpult, die er einem mittlerweile verurteilten Kindermörder „verpassen“ würde. ${ }^{116}$

Äußerst problematisch für die NPD erwiesen sich darüber hinaus die Ermittlungen gegen Mathias Paul Ende 2006 wegen des Verdachts auf Besitz von kinderpornographischen Schriften. Nachdem die Landtags- und Bürgerbüroräume des Verdächtigen durchsucht und umfangreiches Beweismaterial beschlagnahmt worden war, legte Paul sein Mandat nieder und trat zudem von allen Parteiämtern zurück. ${ }^{117}$ Gerade mit der Forderung nach härteren

110 Vgl. Hauen und Stechen, in: Blick nach Rechts, a.a.O., S. 2

111 Vgl. ebenda. Dass Spaltungstendenzen vorhanden sind, zeigt das folgenden Beispiel: Mirko Schmidt hat mittlerweile die „Sächsische Volkspartei“ (SVP) gegründet, die laut seinen Angaben über circa 50 Mitglieder verfügt. Zusammen mit dem früher ebenfalls zur NPD gehörenden Peter Müller gehört er als nun als SVP-Fraktion dem Stadtrat von Meißen an. Im Kreistag des Landkreises Meißen hat er zusammen mit Anke Schmidt und Michael Fleischer (beide ebenfalls Ex-NPD-Mitglieder) auch eine SVP-Fraktion ins Leben gerufen. Vgl. http://nip.systemli.org/ Article251.html [3. Mai 2007].

112 Vgl. Homepage des NPD-Landesverbandes Sachsen, Landesverband Sachsen führt ordentlichen Parteitag durch, http://www.www.sachsen.npd.de [15. März 2006].

113 Vgl. Hauen und Stechen, in: Blick nach Rechts, a.a.O., S. 3.

114 Vgl. Die Welt vom 14. November 2006.

115 Vgl. Ex-NPD-Abgeordneter fliegt aus dem Landtag, auf: Spiegel Online [8. Februar 2007].

116 Vgl. Frankfurter Allgemeine Sonntagszeitung vom 27. Dezember 2006.

117 Vgl. NPD-Abgeordneter legt Mandat nieder, auf: Spiegel Online [8. Februar 2007]. 
Strafen für derartige Vergehen - bis hin zur Todesstrafe für Kindermörder - hatte sich die Partei in diesem populistisch dankbaren Themenfeld zu profilieren versucht. ${ }^{118}$ Allerdings erscheint es dennoch wenig überzeugend, wenn Fraktionschef Apfel für seinen ehemaligen Parteifreund nun die Höchststrafe in dem Verfahren fordert. ${ }^{119}$

Von weit reichender Bedeutung für Fraktion und Partei dürfte sich auch der Unfalltod von Uwe Leichsenring im August 2006 erweisen. ${ }^{120}$ Leichsenring galt vielen Beobachtern als der „Prototyp eines neuen NPD-Repräsentanten“, dessen Äußeres sich einerseits als betont bürgerlich charakterisieren lässt, andererseits aber mit radikalen Einstellungen und Äußerungen auf sich aufmerksam machte. Er unterhielt nicht nur offensichtlich enge Verbindungen zu den verbotenen "Skinheads Sächsische Schweiz"121, sondern begründete und verteidigte in einem Interview auch den verfassungsfeindlichen Charakter der Partei, da diese eine neue Gesellschaftsordnung anstrebe. ${ }^{122}$ Leichsenring erfüllte für die NPD gleich zwei wichtige Funktionen: Ihm gelang es, der Partei das Gesicht einer seriösen „Bürgerpartei“ zu geben, die sich zuallererst um die Belange der Menschen kümmert und deren rechtsextreme Ausrichtung als ein „Schönheitsfehler“ wahrgenommen wird. Gleichzeitig - und im Gegensatz zu den demokratischen Parteien - erreichte er die regionale Jugendszene und konnte diese teilweise sogar einbinden, womit er zusätzlich eine integrative und für das Innenleben der Partei wichtige Rolle übernommen hatte. ${ }^{123}$ Der Verlust von Leichsenring dürfte die Partei somit hart treffen, da sowohl für die Wähler und Sympathisanten als auch für die Mitglieder eine wichtige Identifizierungsfigur verloren gegangen ist.

Zukünftig kann sich die NPD-Fraktion auch aus einem praktischen Grund keine weiteren Abgänge leisten: Nachdem René Despang und Peter Klose für Leichsenring und Paul nachgerückt sind, ist die Landesliste erschöpft. Somit zählt die Fraktion anstatt der ursprünglich zwölf nur noch acht Sitze im Sächsischen Landtag.

\section{Die NPD in Sachsen: besondere Erfolgsbedingungen 2004, Wiederholung und Übertragbarkeit fraglich}

Mit dem Einzug der NPD in den Landtag schien die langjährige Durststrecke der Partei zunächst durchschritten. Neben dem erstmaligen Überwinden der Sperrklausel auf Landesebene seit 36 Jahren konnte sie die Zahl ihrer kommunalen Mandate bundesweit nahezu verdreifachen. Bei den am 13. Juni 2004 gleichzeitig mit der Europawahl stattfindenden Kommunalwahlen in Mecklenburg-Vorpommern, dem Saarland, Sachsen-Anhalt und Sachsen gelang es, insgesamt 62 Sitze zu erringen, drei Monate später in Nordrhein-Westfalen (26. September 2004) entfielen noch einmal zwölf Mandate auf die Partei. Damit verfügt sie nunmehr über 96 Sitze in den Kommunalparlamenten der Republik (vor 2004: 36). Mit einem NPD-Stimmenanteil von 1,6 Prozent bei der Landtagswahl in Thüringen

118 Vgl. Faltblatt: NPD-Landesverband Sachsen (Hrsg.), Todesstrafe für Kinderschänder, Riesa o. J.

119 Vgl. Frankfurter Allgemeine Sonntagszeitung vom 27. Dezember 2006.

120 Vgl. NPD-Politiker stirbt bei Verkehrsunfall, auf: Spiegel Online [21. Februar 2007].

121 Vgl. Franziska Brech, a.a.O., S. 10.

122 Zitiert nach: SPD-Parteivorstand, Wölfe ohne Schafspelz. So sprechen die Rechtsextremen von NPD und DVU, wenn sie keine Kreide gefressen haben, Berlin 2006, S. 9.

123 Vgl. „Daran ist der Uwe schuld“, auf: Spiegel Online [21. Februar 2007]. 
vom Juni 2004 und mit vier Prozent im Saarland im September desselben Jahres konnte man weitere damals achtbare Wahlergebnisse verzeichnen. ${ }^{124}$

Trotzdem ist es offensichtlich nicht gelungen, die Euphorie über diese Erfolge in weitere Wählerstimmen umzuwandeln. Die Parteipresse feierte den Einzug in den Sächsischen Landtag bereits als „Fanal“, von einem „Dammbruch“ war die Rede. Die Partei, die „am längsten und konsequentesten den Kampf gegen das System von 1949 führt“, wolle nun ganz Deutschland erobern. ${ }^{125}$ Dass die Realität allerdings anders aussieht, zeigte alsbald die Landtagswahl in Schleswig-Holstein. Mit einem Resultat von 1,9 Prozent blieb die Partei weit hinter ihren inzwischen gewachsenen Erwartungen zurück. ${ }^{126}$ Im nördlichsten Bundesland fehlte der NPD ein wesentlicher Grund für den Erfolg in Sachsen: Ihre Kandidaten verfügten über keine gesellschaftliche Verankerung. ${ }^{127}$ Wenige Monate später in NordrheinWestfalen (22. Mai 2005) gelang es ebenfalls nicht, an die sächsischen Erfolge anzuknüpfen. Mit 0,9 Prozent der abgegebenen Stimmen verfehlte die Partei sogar die Zulassung zur staatlichen Parteienfinanzierung. ${ }^{128}$ Ebenfalls enttäuschend verlief die vorgezogene Bundestagswahl im September 2005. Zwar erreichte die NPD mit 1,6 Prozent eine Vervierfachung ihres Resultates von 2002, blieb aber von ihrem Ziel, dem Einzug in den „Reichstag“ in Berlin, weit entfernt. ${ }^{129}$ Da die Parteiführung bereits früh die Aussichtslosigkeit ihres Versuchs, bundesweit mehr als fünf Prozent der Stimmen zu erhalten, erkannte, strebte sie in Sachsen den Gewinn von drei Direktmandaten an - ein ebenso unrealistisches Unterfangen, wie das Ergebnis zeigte. ${ }^{130}$ Die Führungsgremien bewerteten das Wahlergebnis dennoch als Erfolg, schließlich sei es das beste auf Bundesebene seit 1969. Zudem sei es gelungen, in jedem Bundesland, mit Ausnahme von Nordrhein-Westfalen, mehr als ein Prozent des Wahlvolkes von sich zu überzeugen. ${ }^{131}$ Diese Befunde können als Indiz dafür gewertet werden, dass sich die sächsischen Erfolge so nicht auf die anderen Bundesländer übertragen lassen.

Der NPD-Landesverband Sachsen hat sich seit seiner Gründung zu der dominanten Gliederung im Bundesverband entwickelt. In bestimmten Gebieten Sachsens, wie dem Erzgebirge oder der Sächsischen Schweiz, verfügt die Partei über eine schlagkräftige Organisation, die das Führen von flächendeckenden Wahlkämpfen ermöglicht. Vor Ort wird die NPD von Vertretern des kleinbürgerlichen Establishments repräsentiert, die ihr den Anstrich einer seriösen und achtbaren Partei geben. Die Bürger nehmen sie nicht als rechtsextrem wahr, den Parteimitgliedern ist es vielmehr durch ehrenamtliche Tätigkeiten und sozialpolitisches Engagement gelungen, die - in den Augen der Bevölkerung - positiven Seiten als Erscheinungsbild dominieren zu lassen. Zudem werden die extremistischen Sicht-

$124 \mathrm{Vgl}$. Bundesministerium des Innern, Verfassungsschutzbericht 2004, Berlin 2005, S. 73 [1. Juni 2005].

125 Deutsche Stimme, Nr. 10/2004.

126 Vgl. Deutsche Stimme, Nr. 03/2005.

127 Vgl. taz vom 19. Februar 2005, S. 5.

128 Vgl. http://www.wahlrecht.de [30. Juli 2005].

129 Vgl. Klartext. Sonderausgabe. Informationen der NPD-Fraktion im Sächsischen Landtag: „Auf dem Weg in die Mitte des Volkes“. Interview mit dem NPD-Fraktionsvorsitzenden im Sächsischen Landtag, Holger Apfel, MdL, S. 3.

$130 \mathrm{Vgl}$. Eckhard Jesse, Das Auf und Ab der NPD, a.a.O., S. 38.

131 Vgl. Udo Voigt, Nach der Wahl ist vor der Wahl. Mit Geschlossenheit zum Erfolg, in: Deutsche Stimme, Nr. 10/2005. 
weisen der Parteiprogrammatik den lokalen politischen Problemen untergeordnet ${ }^{132}$; die NPD zeigt nicht ihr wahres rechtsextremes Gesicht, sondern trägt die Maske einer auf soziale Gerechtigkeit bedachten, kapitalismuskritischen und für Belange der ostdeutschen Bevölkerung kämpfenden „Vertreterin des kleinen Mannes“.

Dabei hat sie ihre Strategie umgestellt. Statt wie früher auf Demonstrationen durch die Straßen zu marschieren, kümmert sie sich gezielt um die Sorgen der „kleinen Leute“ vor Ort. Die Aufmerksamkeit liegt in erster Linie auf der lokalen Jugend, der man Freizeitmöglichkeiten bietet oder indem man systematisch vor Jugendclubs und Schulen wirbt. ${ }^{133}$ Ziel der Partei ist es nicht mehr nur, gute Wahlergebnisse zu erreichen, sondern sich in der „Mitte des Volkes zu etablieren"134. Daher gibt es in einigen Landstrichen des Ostens keinen gesellschaftlichen Widerstand gegen die Partei. ${ }^{135}$ Selbst die ehemalige CDU-Landesregierung ignorierte lange Zeit das Problem und zeichnete das Bild eines „rechtsextremismusfreien" Sachsens. ${ }^{136}$

Zusammenfassend kann jedoch gesagt werden, dass es der NPD im Freistaat insgesamt nicht gelungen ist, sich aus der gewohnten „Außenseiterrolle“ zu befreien, selbst wenn die Erfolge in ihren Hochburgen ein solches Urteil suggerieren. Landesweit lehnt nach wie vor eine große Mehrheit der Bevölkerung die NPD ab, auch wenn fast ein Viertel der Bürger sie bei Befragungen als demokratische Partei wie jede andere einstuft. ${ }^{137}$ Das Ergebnis der letzten Bundestagswahl, bei der die Partei auch in Sachsen mit 4,8 Prozent an der Sperrklausel scheiterte, unterstreicht diese Feststellung. Dennoch muss davon ausgegangen werden, dass sich dort in den letzten Jahren ein Stammwählerpotenzial etabliert hat, das besonders aus rechtsextrem eingestellten jungen Männern besteht. Dieses Potenzial allein reicht indes nicht aus, um die Fünfprozenthürde zu überschreiten. Hinzu kommen die NPDStammwähler aus den Hochburgen, die für die Partei wahrscheinlich nicht primär wegen der Programmatik, sondern aufgrund ihrer Vertreter stimmen.

Zusätzlich zu ihrer strukturell-gesellschaftlichen Verankerung in bestimmten Gebieten Sachsens begünstigte die aktuelle politische Situation vor der Landtagswahl die Erfolgsaussichten der NPD. Auch die Debatte um die Arbeitslosigkeit (symbolisiert durch „Hartz IV“), der Vertrauensverlust der Volksparteien sowie die Unzufriedenheit mit der Arbeit der Bundesregierung motivierten ein nicht unbeträchtliches Protestpotenzial zur Stimmabgabe für die Rechtsextremen. ${ }^{138}$ Daneben spielt die Auflösung der traditionellen Wählermilieus eine Rolle. In den neuen Bundesländern ist die Wählerschaft deutlich anders zusammengesetzt als im Westen. Naturgemäß konnten zu Zeiten der ehemaligen DDR die Bürger keine enge Bindung zu den Parteien (mit Ausnahme der PDS) aufbauen. ${ }^{139}$ Dies begünstigt den Typus des Wechselwählers, der durchaus sein Kreuz auch bei den Rechtsparteien machen

132 Vgl. Reportage „Lauter nette Leute“ auf Phoenix (5. März 2005).

133 Vgl. Der Spiegel, Nr. 40/2004 [6. Januar 2005].

134 Vgl. Klartext. Sonderausgabe, a.a.O., S. 3.

135 Vgl. Toralf Staudt, a.a.O., S. 130.

136 Vgl. ebenda, S. 140.

137 Vgl. Infratest dimap (Hrsg.), a.a.O., S. 86.

138 Vgl. Viola Neu, a.a.O.

139 Vgl. Ulrich von Alemann, Parteien und Gesellschaft in der Bundesrepublik. Rekrutierung, Konkurrenz und Responsivität, in: Alf Mintzel / Heinrich Oberreuter (Hrsg.), Parteien in der Bundesrepublik Deutschland, 2., aktualisierte und erweiterte Ausgabe, Bonn 1992, S. 89 - 130, S. 106 . 
kann. Als letzter Erfolgsgrund für die sächsische NPD kann ihr Monopol in der rechten Parteienlandschaft genannt werden. Gemäß einer Wahlabsprache trat die DVU nicht an und riet ihren Wählern, für die NPD zu stimmen. Auch die REP und die DP, die bei den Europawahlen wenige Monate zuvor der NPD noch Konkurrenz machen konnten, standen am 19. September 2004 nicht auf dem Stimmzettel. Bedacht werden muss außerdem, dass in den neuen Bundesländern der latente Rechtsextremismus in der Bevölkerung stärker verbreitet ist als im Westteil des Landes. ${ }^{140}$ Wie die empirische Wahlforschung zeigen konnte, erhöht eine derartige Einstellung die Bereitschaft zur Wahl einer Partei der extremen Rechten. Damit verfügt die NPD auf dem Gebiet der ehemaligen DDR - zumindest theoretisch - über ein erhöhtes Mobilisierungspotenzial, welches so in keinem westdeutschen Bundesland vorliegt.

Damit waren die Erfolgsbedingungen für die NPD bei der sächsischen Landtagswahl ideal. Eine derartige Sondersituation wie im September 2004 in Sachsen ist bundesweit kein zweites Mal vorhanden. ${ }^{141}$ Die Partei konnte sich als alleinige nationale Oppositionspartei mit gesellschaftlicher Verankerung und mit Kandidaten aus „der Mitte der Gesellschaft" präsentieren; zudem spielte ihr die politische Großwetterlage mit der Debatte um „Hartz IV“ offensichtlich in die Hände. Vereinfacht könnte man sagen: Die NPD war zur richtigen Zeit am richtigen Ort.

Dennoch kann der Partei in Sachsen wegen ihrer organisatorischen und strategischen Entwicklung eine „Vorreiterrolle“ innerhalb der bundesrepublikanischen extremen Rechten bescheinigt werden. Die sächsische NPD verfügt über eine herausragende Stellung auf dem rechten Flügel des regionalen Parteiensystems. Ihr ist es gelungen, neben dem neonationalsozialistischen Teil der Szene auch die bis dato konkurrierenden nationalen Parteien für eine Zusammenarbeit zu gewinnen. ${ }^{142}$

140 Vgl. beispielsweise: Corinna Kleinert / Johann de Rijke, Rechtsextreme Orientierung bei Jugendlichen und jungen Erwachsenen, in: Wilfried Schubarth / Richard Stöss (Hrsg.), Rechtsextremismus in der Bundesrepublik Deutschland. Eine Bilanz, Bonn 2000, S. 167 - 198, S. 185.

141 Vgl. taz vom 13. Oktober 2004, S. 3.

142 Vgl. Toralf Staudt, a.a.O., S. 58. 